\title{
Rao-Blackwellized Free Clustering Particle PHD Filter For Multi-Target Tracking
}

\author{
Ping YE ${ }^{1, a}$, Hui $\mathrm{CHEN}^{1, b}$ \\ ${ }^{1}$ School of Electrical \& Information Engineering, Lanzhou University of Technology, Lanzhou, China \\ ashockwavealex@163.com, bhuich78@hotmail.com
}

Keywords: Rao-Blackwellized Particle Filter, Free Clustering, Particle PHD Filter, Kalman Filter, Linear Component, Non Linear Component, Multi-Target Tracking

\begin{abstract}
This paper presents a novel approach to improve the accuracy of auxiliary particle probability hypothesis density filter without the need of clustering step. In most cases, the states of target can be seen as combinations of linear components and non linear ones. The new approach separates the two different components and then adopts auxiliary particle filter (APF) and Kalman filer to estimate the non linear parts and linear parts. After the update step it extracts the multi-target states via the predict particles and their updated weights which correspond to each measurement. The simulation results illustrate the effectiveness of the presented approach.
\end{abstract}

\section{Introduction}

The aim of multi-target tracking (MTT) [1] is to estimate the states of targets from a set of observations with uncertainty. It is a difficulty in the field of target tracking. On the target side the number of target is usually unknown in the whole process, any target can appear or disappear at any moment. One target may spawn when it is moving. On the other hand, the sensor is not able to detect all targets at one moment, and sometimes irrelevant objects could be treated as targets in a scanning cycle, so inevitably many false detections are mixed in the observations. The possibilities above increase the complexity of MTT problem. Before the application of Finite Set Statistics (FISST) the classical approaches such as Multi-hypothesis Tracking (MHT) [2] and Joint Probabilistic Data Association filter (JPDAF) [3] were widely utilized to solve such difficulties. However, the shortcomings of these approaches are also obvious because they require the knowledge of the expected number of targets, and the data correlation process determines their performance directly. In order to avoid the data correlation process Mahler proposed the FISST and it has been proven to be an effective way especially when the number of targets is unknown. To reduce the complexity further, the Probability Hypothesis Density (PHD) filter was proposed by Mahler [4]. It is an approximate alternative filter to the optimal Bayesian multi-target filter. PHD filter predicts and updates the first moment of the multi-target posterior constantly to get the number of targets at each moment. Many implementations of this filter have been proposed, Particle Filtering (PF) approach [5-7] is one of the most widely used methods.

PF provides a valid solution for the estimation of posterior when the system state is non linear. However, when the dimensions of system state increase, the computational complexity of PF also increases with it, this could cost huge amount of computational resource and time. In many applications not all the dimensions of target state are non linear. In this case the Rao-Blackwell theorem provides a solution for reducing the computational complexity of PF. A typical mixed algorithm is called Rao-Blackwellized Particle filter (RBPF). RBPF combines Kalman filter (KF) with PF, thus it creates a parallel filter banks. It uses PF to estimate the non linear components and KF to deal with the linear ones. A lot of research have been done to develop RBPF filters. With respect to the PF, RBPF can lower the dimensions of system and increase the estimation accuracy[8,9,10,11,12].

Though PF has been proven to be an effective method to implement PHD filter, calculating the states of targets from a set of particles is a difficulty for Particle-PHD (P-PHD, also known as 
SMC-PHD) algorithm. In most cases the K-Means clustering method is widely applied[13,14], however, it usually takes a lot time for K-Means to locate the clustering center and extract the peaks, and the clustering performance is poor. In order to avoid the clustering step Ristic proposed a free clustering method to implement PHD algorithm through auxiliary particle filtering (APF) implementation [15], the results show that this new algorithm improves accuracy of P-PHD greatly.

The main contribution of this paper is to present a novel implementation of PHD filter by applying $\mathrm{RBPF}$ in the existing free clustering P-PHD filter to track multi-target. In section 2 we briefly recall the random finite set and the PHD filter. In section 3 the concrete implementation steps of the presented approach is introduced. We compare the performance of the presented approach with 2 other improved P-PHD filters, with and without K-Means clustering method in section 4. In section 5 we draw a conclusion according to the simulation and end the paper with it.

\section{Background}

\subsection{Multi-target state and measurement model}

The state of an individual target at moment $k$ can be described by set $\mathbf{X}$ in state space $\mathcal{X}$. Assume that at moment $k$ there are $N(k)$ targets scattering in state space, and each of them is located at $\mathbf{x}_{k, 1}, \ldots, \mathbf{x}_{k, N(k)}$, then the multi-target state are represented by the following finite set:

$$
\mathbf{X}_{k}=\left\{\mathbf{x}_{k, 1}, \ldots, \mathbf{x}_{k, N(k)}\right\} \in F(\mathcal{X}) .
$$

Similarly, the measurement of individual target at moment $k$ can be described by set $\mathbf{z}$ in measurement space $\mathcal{Z}$. At moment $k$ the sensor reports $M(k)$ measurements $\mathbf{z}_{k, 1}, \ldots, \mathbf{z}_{k, M(k)}$, then the measurement set can be written as:

$$
\mathbf{Z}_{k}=\left\{\mathbf{z}_{k, 1}, \ldots, \mathbf{z}_{k, M(k)}\right\} \in F(\mathcal{Z}) .
$$

In the process of state transition any target may appear at any moment, meanwhile the survival targets may disappear. So the number of target is always uncertain. As for the measurements, since not all existing targets at each moment will be detected, and the observation set may include false detections. The uncertainties above cause the number of elements of both sets $\mathbf{X}_{k}$ and $\mathbf{Z}_{k}$ are variables over time. They are also called random finite set (RFS).

The RFS of the multi-target state $\mathbf{X}_{k}$ at moment $k$ is represented by the union

$$
\mathbf{X}_{k}=\left(\cup_{\mathbf{x} \in \mathbf{X}_{k-1}} S_{k \mid k-1}(\mathbf{x})\right) \cup \Gamma_{k},
$$

where $S_{k \mid k-1}(x)$ is the RFS of targets which survive from moment $k-1$ to $k, \Gamma_{k}$ is the birth targets which appear at moment $k$. The RFS measurements can be represented as

$$
\mathbf{Z}_{k}=\left[\cup_{\mathbf{x} \in \mathbf{x}_{k}} \Theta_{k}\left(\mathbf{x}_{k}\right)\right] \cup \kappa_{k},
$$

where $\Theta_{k}\left(\mathbf{x}_{k}\right)$ is the RFS of measurements that sensor reports at moment $k, \kappa_{k}$ represents the false detections at moment $k$.

\subsection{The PHD filter}

PHD is the first-order moment of the posterior distribution of RFS $\mathbf{X}_{k}$. The integral value of the PHD over a specific region in the state space is the expected number of targets within this region. Like the multi-target Bayesian filter, the PHD recursion consists of prediction and update step. Assume $v_{k \mid k}\left(\mathbf{x}_{k}\right)$ is the PHD which associated with the posterior $p_{k \mid k}\left(\mathbf{X}_{k} \mid \mathbf{Z}_{1: k}\right)$, then the prediction step of PHD filter can be written as :

$$
v_{k \mid k-1}\left(\mathbf{x}_{k}\right)=\Gamma\left(\mathbf{x}_{k}\right)+\int\left[p_{S}\left(\mathbf{x}_{k-1}\right) f\left(\mathbf{x}_{k} \mid \mathbf{x}_{k-1}\right)+\Gamma\left(\mathbf{x}_{k} \mid \mathbf{x}_{k-1}\right)\right] v_{k-1 \mid k-1}\left(\mathbf{x}_{k-1}\right) d\left(\mathbf{x}_{k-1}\right),
$$


where $f\left(\mathbf{x}_{k} \mid \mathbf{x}_{k-1}\right)$ is the transition density of multi-target, $p_{s}\left(\mathbf{x}_{k-1}\right)$ is the probability which target survive from moment $k-1$ to $k, \Gamma\left(\mathbf{x}_{k} \mid \mathbf{x}_{k-1}\right)$ represents the intensity function of RFS of targets spawned from $k-1$ to $k$, and $\Gamma\left(\mathbf{x}_{k}\right)$ is the intensity function of birth of new targets. Then the update step of PHD filter can be represented as

$$
v_{k \mid k}\left(\mathbf{x}_{k}\right)=v_{k \mid k-1}\left(\mathbf{x}_{k}\right)\left(1-p_{D}\left(\mathbf{x}_{k}\right)\right)+\sum_{\mathbf{z} \in \mathbf{Z}_{k}} \frac{v_{k \mid k-1}\left(\mathbf{x}_{k}\right) p_{D}\left(\mathbf{x}_{k}\right) g\left(\mathbf{z} \mid \mathbf{x}_{k}\right)}{\lambda c(\mathbf{z})+\int p_{D}\left(\mathbf{x}_{k}\right) g\left(\mathbf{z} \mid \mathbf{x}_{k}\right) v_{k \mid k-1}\left(\mathbf{x}_{k}\right) d\left(\mathbf{x}_{k}\right)},
$$

where $p_{D}\left(\mathbf{x}_{k}\right)$ is probability of detection of state $\mathbf{x}_{k}, \lambda$ is the average number of false detections each moment and $c(\mathbf{z})$ is the probability distribution of every false detection, $g\left(\mathbf{z} \mid \mathbf{x}_{k}\right)$ is the measurement likelihood. The integral of $v_{k \mid k}\left(\mathbf{x}_{k}\right)$ over a region $S$ is the expected number of targets in $S$.

\section{The Rao-Blackwellized Free Clustering P-PHD Filter}

\subsection{Recall of Rao-Blackwellized particle filtering}

In practical applications the dimension of multi-target state is higher than that of measurement. In this situation the multi-target state $\mathbf{X}_{k}$ can be divided into non linear part $\mathbf{X}_{k}^{n}$ and linear part $\mathbf{X}_{k}^{l}$, their dimensions are $m^{n}$ and $m^{l}$, then the state $\mathbf{X}_{k}$ can be written as $\mathbf{x}_{k}=\left[\mathbf{x}_{k}^{n}, \mathbf{x}_{k}^{l}\right]^{T}$. Respectively, the state transition equations of the two components are as follows:

$$
\begin{aligned}
& \mathbf{x}_{k}^{n}=f_{k}^{n}\left(\mathbf{x}_{k-1}^{n}\right)+\mathbf{F}_{k}^{n} \mathbf{x}_{k-1}^{l}+\mathbf{w}_{k}^{n}, \\
& \mathbf{x}_{k}^{l}=f_{k}^{l}\left(\mathbf{x}_{k-1}^{n}\right)+\mathbf{F}_{k}^{l} \mathbf{x}_{k-1}^{l}+\mathbf{w}_{k}^{l},
\end{aligned}
$$

where $f_{k}^{n}(\cdot)$ is a function from $\operatorname{IR}^{m^{n}}$ to $\operatorname{IR}^{m^{n}}, \mathbf{F}_{k}^{n}$ is a $m^{n} \times m^{l}$ matrix which is connected with the parts $\mathbf{x}_{k-1}^{l}$ and acts on $\mathbf{x}_{k-1}^{n}, f_{k}^{l}$ and $\mathbf{F}_{k}^{l}$ are defined similarly. The measurement equation can be written as:

$$
\mathbf{z}_{k}=h_{k}\left(\mathbf{x}_{k}^{n}\right)+\mathbf{v}_{k}^{n}
$$

The non linear measurement this paper mainly focuses on is Rang-Bearing tracking, then $h_{k}$ is:

$$
h_{k}(\mathbf{x})=\left[\arctan (\mathbf{y} / \mathbf{x}), \sqrt{\mathbf{x}^{2}+\mathbf{y}^{2}}\right]^{T} .
$$

The connection between their noise vector is as follows:

$$
\left[\begin{array}{c}
\mathbf{w}_{k}^{n} \\
\mathbf{w}_{k}^{l} \\
\mathbf{v}_{k}^{n}
\end{array}\right] \sim \mathcal{N}\left(;\left[\begin{array}{l}
0 \\
0 \\
0
\end{array}\right] ;\left[\begin{array}{ccc}
\mathbf{Q}_{k}^{n} & \mathbf{Q}_{k}^{n l} & 0 \\
\left(\mathbf{Q}_{k}^{n l}\right)^{T} & \mathbf{Q}_{k}^{l} & 0 \\
0 & 0 & \mathbf{R}_{k}
\end{array}\right]\right),
$$

where $\mathcal{N}\left(0, \sigma^{2}\right)$ means Gaussian distribution with zero mean and variance $\sigma$, the $\left[\mathbf{w}_{k}^{n}, \mathbf{w}_{k}^{l}\right]$ and $\mathbf{v}_{k}^{n}$ at all moments are independent.

When all the state components of system are non linear, the posterior probability density $p\left(\mathbf{X}_{k} \mid \mathbf{Z}_{1: k}\right)$ can be calculated via PF. When the state equation can be separated as previous situation, according to the Bayesian theorem, $\mathbf{X}_{k}^{l}$ can be separated from $p\left(\mathbf{X}_{k} \mid \mathbf{Z}_{1: k}\right)$ and thereby be calculated separately, then $p\left(\mathbf{X}_{k} \mid \mathbf{Z}_{1: k}\right)$ can be written as:

$$
p\left(\mathbf{X}_{k}^{l}, \mathbf{X}_{k}^{n} \mid \mathbf{Z}_{1: k}\right)=p\left(\mathbf{X}_{k}^{l} \mid \mathbf{X}_{k}^{n}, \mathbf{Z}_{1: k}\right) p\left(\mathbf{X}_{k}^{n} \mid \mathbf{Z}_{1: k}\right),
$$

in which $p\left(\mathbf{X}_{k}^{n} \mid \mathbf{Z}_{1: k}\right)$ is calculated via PF, and $p\left(\mathbf{X}_{k}^{l} \mid \mathbf{X}_{k}^{n}, \mathbf{Z}_{1: k}\right)$ can be computed via KF in the sense of minimum variance. 


\subsection{Implementation of Rao-Blackwellized Free Clustering P-PHD Filter}

There are three main drawbacks in P-PHD algorithm. First, it needs to spread large amount of particles in the state space because the probability of targets appear anywhere in it is equal, the particles together represent the birth density. This increases the consumptions of computation resources. Second, after update step, P-PHD has to cluster all the updated particles via K-Means technique, the purpose is to make every particle correspond to its target and get state estimation values from different particle swarms. However, the precision cannot be guaranteed. Third one is that the number of target estimation gained from the particles' weights is unstable. To overcome these disadvantages, Ristic proposed an APF based approach to implement P-PHD. In his method, the birth density is described by a bunch of particles generated by measurement at each moment, so the filter does not need to spread particles in state space where targets do not appear, this undoubtedly reduces the number of particles, and thus save the running time. In the update step, the filter obtains state estimations directly from updated weights of all particles, their values change correspond to each measurement. This increases the accuracy of state estimation. Since the participations of measurement generated newborn particles, the cardinality estimation is more stable compare to P-PHD.

The following section introduces the approach to plug RBPF into the improved P-PHD algorithm which introduced above, so as to present a new implementation of P-PHD filter.

\subsubsection{Prediction:}

At the moment $k$, in APF based P-PHD filter, the input persistent particle set is described by $\left\{\mathbf{w}_{p, k-1}^{i}, \mathbf{x}_{p, k-1}^{i}\right\}_{i=1}^{P_{k-1}}$, where $P_{k-1}=N_{p} n_{k-1}$ is the number of all persistent particles, $N_{p}$ is the number of persistent particles for each target, $n_{k-1}$ is the estimated number of target number from last moment. The newborn particle set from $k-1$ is generated by measurements, they can be written as $\left\{\mathbf{w}_{b, k-1}^{i}, \mathbf{x}_{b, k-1}^{i}\right\}_{i=1}^{B_{k-1}}, \mathbf{w}_{b, k-1}^{i}$ can be computed according to Eq. 28, $B_{k-1}=N_{b} m_{k-1}$ is the number of all newborn particles, $N_{b}$ is the number of newborn particles generated from each measurement, $m_{k-1}$ is the number of measurement. Then merge the two particle set to one, we can get the persistent particle set:

$$
\left\{\mathbf{w}_{k-1}^{i}, \mathbf{x}_{k-1}^{i}\right\}_{i=1}^{I_{k-1}}=\left\{\mathbf{w}_{p, k-1}^{i}, \mathbf{x}_{p, k-1}^{i}\right\}_{i=1}^{P_{k-1}} \cup\left\{\mathbf{w}_{b, k-1}^{i}, \mathbf{x}_{b, k-1}^{i}\right\}_{i=1}^{B_{k-1}},
$$

then the persistent PHD $v_{k-1 \mid k-1}\left(\mathbf{x}_{k-1}\right)$ can be described by their weights:

$$
v_{k-1 \mid k-1}\left(\mathbf{x}_{k-1}\right) \approx \sum_{i=1}^{L_{k-1}} \mathbf{w}_{k-1}^{i} \delta_{\mathbf{x}_{k-1}^{i}}\left(\mathbf{x}_{k-1}\right) \text {, }
$$

where $\delta_{\mathbf{x}_{k-1}^{i}}\left(\mathbf{x}_{k-1}\right)$ is the Dirac delta function concentrated at the value $\mathbf{x}_{k-1}^{i}$. Since every particle contains linear part and non linear part, the persistent particles $\mathbf{x}_{k-1}^{i}$ can be decomposed as $\mathbf{x}_{k-1}^{i}=\left[\mathbf{x}_{k-1}^{n, i}, \mathbf{x}_{k-1}^{l, i}\right]$. Plug $\mathbf{x}_{k-1}^{n, i}$ into the state transition Eq. 7 and get $\mathbf{x}_{k \mid k-1}^{n, i}$, the weights $\mathbf{w}_{k-1}^{i}$ change as follows:

$$
\mathbf{w}_{k \mid k-1}^{i}=p_{S}\left(\mathbf{x}_{k \mid k-1}^{i}\right) \mathbf{w}_{k-1}^{i},
$$

where $p_{s}\left(\mathbf{x}_{k \mid k-1}^{i}\right)$ is survival probability for each particle. Since the linear part $\mathbf{x}_{k-1}^{l, i}$ corresponds to linear Gaussian system, the predicted PHD becomes:

$$
v_{k \mid k-1}\left(\mathbf{x}_{k \mid k-1}^{i}\right) \approx \sum_{i=1}^{L_{k-1}} \mathbf{w}_{k \mid k-1}^{i} \delta_{\mathbf{x}_{k-1}^{n, i}}\left(\mathbf{x}_{k}^{n}\right) \mathcal{N}\left(\mathbf{x}_{k \mid k-1}^{l, i} ; \mathbf{P}_{k}^{l, i}\right),
$$

where $\mathbf{x}_{k \mid k-1}^{l, i}$ is the average value of linear part, $\mathbf{P}_{k \mid k-1}^{l, i}$ is the relevant covariance. The two parameters are obtained via KF, their calculation formulas are as follows:

$$
\mathbf{A}_{k}=\mathbf{F}_{k}^{l, i}-\mathbf{Q}_{k}^{n l}\left(\mathbf{Q}_{k}^{n}\right)^{-1} \mathbf{F}_{k}^{n, i}
$$




$$
\begin{aligned}
& \mathbf{K}_{k}=\mathbf{P}_{k-1}^{l, i}\left(\mathbf{F}_{k}^{n, i}\right)^{T}\left[\mathbf{F}_{k}^{n, i} \mathbf{P}_{k-1}^{l, i}\left(\mathbf{F}_{k}^{n, i}\right)^{T}+\mathbf{Q}_{k}^{n}\right]^{-1}, \\
& \mathbf{P}_{k}^{l, i}=\mathbf{Q}_{k}^{l}-\mathbf{Q}_{k}^{n l}\left(\mathbf{Q}_{k}^{n}\right)^{-1}\left(\mathbf{Q}_{k}^{n l}\right)^{T}+\mathbf{A}_{k}\left[\left(\mathbf{I}-\mathbf{K}_{k} \mathbf{F}_{k}^{n, i}\right) \mathbf{P}_{k-1}^{l, i}\right]\left(\mathbf{A}_{k}\right)^{T}, \\
& \tilde{\mathbf{x}}_{k \mid k-1}^{,, i}=\mathbf{x}_{k-1 \mid k-1}^{l, i}+\mathbf{K}_{k}\left(\mathbf{x}_{k \mid k-1}^{n, i}-\mathbf{F}_{k}^{n, i} \mathbf{x}_{k-1 \mid k-1}^{l, i}\right), \\
& \mathbf{x}_{k \mid k-1}^{l, i}=\mathbf{A}_{k} \tilde{\mathbf{x}}_{k \mid k-1}^{l, i}+f_{k}^{l, i} \mathbf{x}_{k-1 \mid k-1}^{n, i}+\mathbf{Q}_{k}^{n l}\left(\mathbf{Q}_{k}^{n}\right)^{-1}\left(\mathbf{x}_{k \mid k-1}^{n, i}-f_{k}^{n, i} \mathbf{x}_{k-1 \mid k-1}^{n, i}\right),
\end{aligned}
$$

where $\mathbf{F}_{k}^{l, i}, f_{k}^{l, i}, \mathbf{F}_{k}^{n, i}, f_{k}^{n, i}$ are defined in section 3.1. Once the linear component $\mathbf{x}_{k \mid k-1}^{l, i}$ and its covariance $\mathbf{P}_{k}^{l, i}$ are obtained, merge $\mathbf{x}_{k \mid k-1}^{l, i}$ together with $\mathbf{x}_{k \mid k-1}^{n, i}$ and get the predicted persistent particle set $\left\{\mathbf{w}_{k \mid k-1}^{i}, \mathbf{x}_{k \mid k-1}^{i}\right\}_{i=1}^{L_{k-1}}$, where $\mathbf{x}_{k \mid k-1}^{i}=\left[\mathbf{x}_{k \mid k-1}^{n, i}, \mathbf{x}_{k \mid k-1}^{l, i}\right]$.

\subsubsection{Update:}

In update step, the weights of persistent particles are updated according to Eq. 22:

$$
\mathbf{w}_{k \mid k}^{i}=\mathbf{w}_{k \mid k-1}^{i}\left(1-p_{D}\left(\mathbf{x}_{k \mid k-1}^{i}\right)\right)+\sum_{\mathbf{z} \in \mathbf{Z}_{k}} \frac{\mathbf{w}_{k \mid k-1}^{i} g_{k}\left(\mathbf{z} \mid \mathbf{x}_{k \mid k-1}^{i}\right) p_{D}\left(\mathbf{x}_{k \mid k-1}^{i}\right)}{\lambda c(\mathbf{z})+\sum_{i=1}^{L_{k-1}} \mathbf{w}_{k \mid k-1}^{i} g_{k}\left(\mathbf{z} \mid \mathbf{x}_{k \mid k-1}^{i}\right) p_{D}\left(\mathbf{x}_{k \mid k-1}^{i}\right)},
$$

where $i=1, \ldots, L_{k-1}, p_{D}\left(\mathbf{x}_{k \mid k-1}^{i}\right)$ is the detection probability for each particle, $g_{k}\left(\mathbf{z} \mid \mathbf{x}_{k \mid k-1}^{i}\right)$ is the likelihood function, $\lambda$ and $c(\mathbf{z})$ are introduced previously. For every measurement, compute the updated weight correspond to each one of them:

$$
\mathbf{w}_{k \mid k}^{i, j}=\frac{\mathbf{w}_{k \mid k-1}^{i} g_{k}\left(\mathbf{z}_{j} \mid \mathbf{x}_{k \mid k-1}^{i}\right) p_{D}\left(\mathbf{x}_{k \mid k-1}^{i}\right)}{\lambda c\left(\mathbf{z}_{j}\right)+\sum_{i=1}^{L_{k-1}} \mathbf{w}_{k \mid k-1}^{i} g_{k}\left(\mathbf{z}_{j} \mid \mathbf{x}_{k \mid k-1}^{i}\right) p_{D}\left(\mathbf{x}_{k \mid k-1}^{i}\right)},
$$

where $j=1, \ldots, m_{k}$. Once $\mathbf{w}_{k \mid k}^{i, j}$ is obtained, for each measurement $j$, compute the following sum

$$
\mathbf{W}_{j}=\sum_{i=1}^{L_{k-1}} \mathbf{w}_{k \mid k}^{i, j} .
$$

If $\mathbf{w}_{j}>\eta$, then it can be considered as valid, the threshold $\eta$ in this paper is 0.8 . To all the valid $\mathbf{w}_{j}$, state estimation is computed as

$$
\hat{\mathbf{x}}_{k, j}=\sum_{i=1}^{L_{k-1}} \mathbf{w}_{k \mid k}^{i, j} \mathbf{x}_{k \mid k-1}^{i} .
$$

The sum of $\mathbf{w}_{k \mid k}^{i}$ is the expected number of current target number estimation

$$
N_{k}=\sum_{i=1}^{L_{k-1}} \mathbf{w}_{k \mid k}^{i} .
$$

Compute the round numbers $n_{k}$ near $N_{k}$, its product with $N_{p}$ is the number of resampled particles. Any resampling algorithm can be used here to get new persistent particle set $\left\{\mathbf{w}_{p, k}^{i}, \mathbf{x}_{p, k}^{i}\right\}_{i=1}^{P_{k}}$. Generate $B_{k}=N_{b} m_{k}$ newborn particles $\left\{\mathbf{w}_{b, k \mid k-1}^{i}, \mathbf{x}_{b, k \mid k-1}^{i}\right\}_{i=1}^{B_{k}}$ from the current measurements, where $\mathbf{w}_{b, k \mid k-1}^{i}=1 / N_{b} \cdot m_{k}$, they can be updated as:

$$
\mathbf{w}_{b, k \mid k}^{i}=\sum_{\mathbf{z} \in \mathbf{Z}_{k}} \frac{\mathbf{w}_{b, k \mid k-1}^{i}}{\lambda c(\mathbf{z})+\sum_{i=1}^{B_{k}} \mathbf{w}_{b, k \mid k-1}^{i}+\sum_{i=1}^{L_{k-1}} \mathbf{w}_{k \mid k-1}^{i} g_{k}\left(\mathbf{z} \mid \mathbf{x}_{k \mid k-1}^{i}\right) \operatorname{Pd}\left(\mathbf{x}_{k \mid k-1}^{i}\right)} .
$$

Then resample from the set $\left\{\mathbf{w}_{b, k \mid k}^{i}, \mathbf{x}_{b, k \mid k-1}^{i}\right\}_{i=1}^{B_{k}}$ to get new newborn particle set $\left\{\mathbf{w}_{b, k}^{i}, \mathbf{x}_{b, k}^{i}\right\}_{i=1}^{B_{k}}$, where 


$$
\mathbf{w}_{b, k}^{i}=\frac{\mathbf{w}_{b, k \mid k}^{i}}{\sum_{i=1}^{B_{k}} \mathbf{w}_{b, k \mid k}^{i}} .
$$

\section{Simulations}

\subsection{Simulation Setup}

This section compares the tracking performance of the presented approach with two APF based P-PHD filters, with and without K-Means clustering step (they are respectively referred to as RBAP-PHD, AP-K-MEANS-PHD and AP-PHD for convenience sake in this section). The range of tracking scenario is set as $[-1000,1000] \times[-1000,1000] \mathrm{m}^{2}$, the sensor is still and located at origin of coordinates. Sampling period is $T=1 \mathrm{~s}$, the total sampling number is 50 . The measurement noise is Gaussian white noise with zero mean, measurement noise covariance is $\sum \operatorname{diag}\left[\sigma_{b}^{2}, \sigma_{r}^{2}\right]$, where $\sigma_{b}=0.1^{\circ}, \sigma_{r}=2 m$ are standard deviations of Range-Bearing measurement. The probability of detection is $p_{D}=0.98$, the survival probability is $p_{s}=0.98$. The number of clutters per scan is 5 , clutter density is $\lambda_{c}=7.96 \times 10^{-4}(\mathrm{rad} \cdot \mathrm{m})^{-1}$. The number of particles for tracking each target is $\mathrm{N}_{p}=100$, every new measurement generates $N_{b}=10$ newborn particles. OSPA distance technique is used to compare the tracking accuracy of filters, it can evaluate both the state estimation and cardinality estimation jointly. Its parameters are $c=200 \mathrm{~m}, p=1$. The software and hardware conditions for experiment is MATLAB 2012b, Windows 7, AMD A8-6500 3.5GHz, RAM 4GB. Assume 7 targets are moving in the state space, they appear and disappear at different moment. The models of their tracks are NCVM. State transform matrix and noise covariance matrix are

$$
\mathbf{F}=\left[\begin{array}{ll}
1 & \mathrm{~T} \\
0 & 1
\end{array}\right] \otimes \mathbf{I}_{2}, \quad \mathbf{Q}=\varpi\left[\begin{array}{cc}
\frac{\mathrm{T}^{3}}{3} & \frac{\mathrm{T}^{2}}{2} \\
\frac{\mathrm{T}^{2}}{2} & \mathrm{~T}
\end{array}\right] \otimes \mathbf{I}_{2},
$$

where $\otimes$ is Kronecker product, $\varpi=0.05$.

\subsection{Experiments}
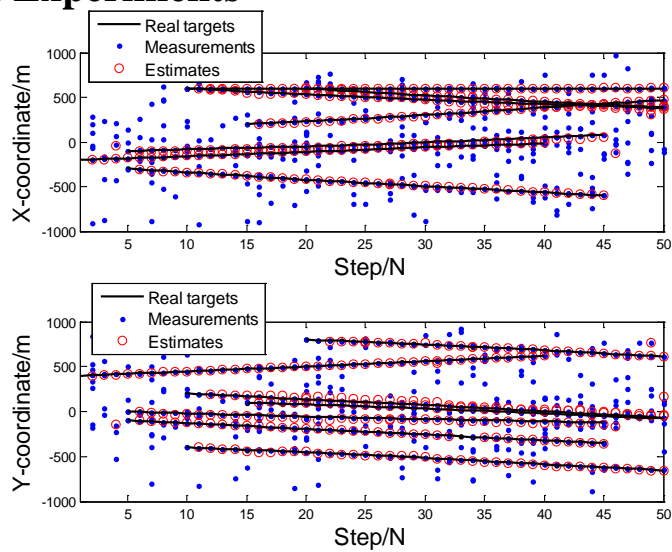

Fig.1. Ground truth: X and Y components of real targets, measurements and output of presented algorithm against time

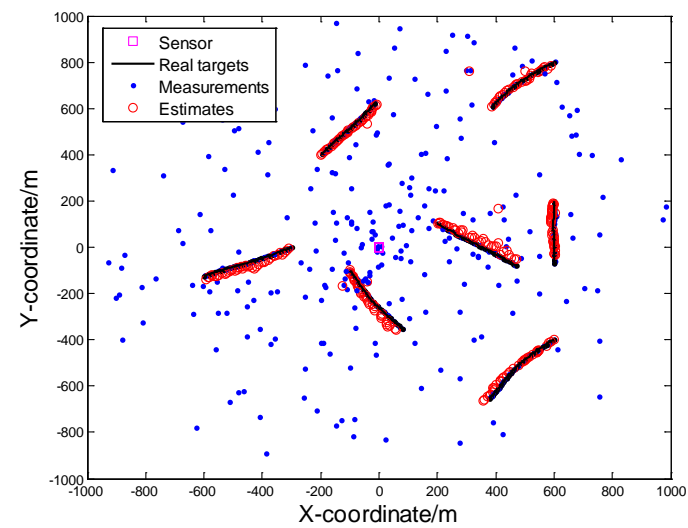

Fig.2. Ground truth: positions of real targets, measurements and output of presented algorithm

Fig.1 and Fig.2 illustrate the output of the presented approach compare to the real multi-target tracks. We can see the estimation points are scattered close to the real tracks. Though at some moments the filter has no output for some individual targets, this may caused by the imperfection of the sensor and the algorithm itself, the overall tracking effect is good, and the filter can track targets at different positions in state space. 


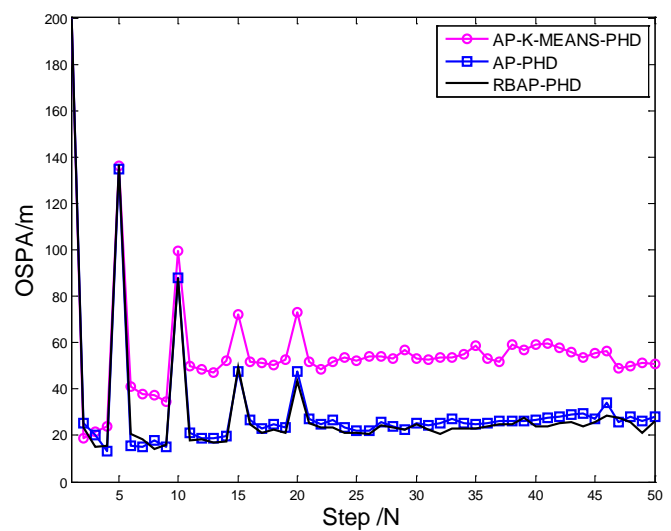

Fig.3. Tracking performance comparison for position OSPA

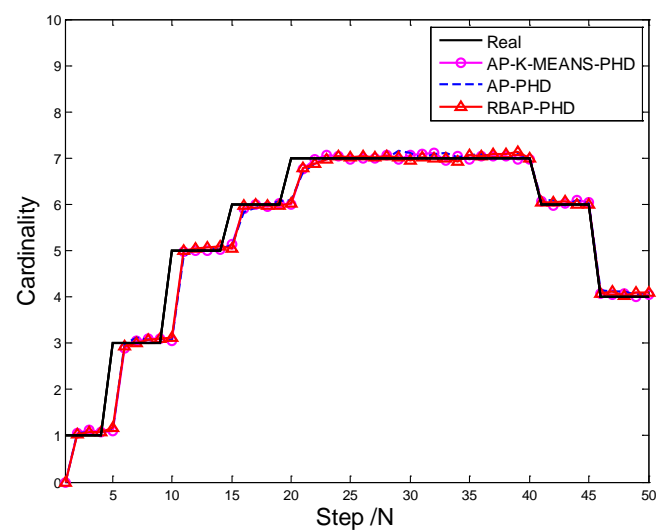

Fig.4. Tracking performance of cardinality estimate

Fig.3 and Fig.4 are results over 100 Monte Carlo runs. Fig.3 shows the OSPA distance comparisons for the three filters. Both the AP-PHD and RBAP-PHD outperform the AP-K-MEAN-PHD. This is because the two filters obtain estimates from the updated weights of persistent particles, and the values of these weights change correspond to all the measurements at each moment, so the individual estimate is distributed to its own target automatically. The result of this approach is much more precise than that of K-Means clustering algorithm. Other than that, the black solid line is lower than the blue one at most moments, meaning RBAP-PHD is more accurate than AP-PHD. It's because RBAP-PHD does Kalman filtering to linear parts of every particles, this makes particles scatter more concentrated near the real targets, hence the OSPA distance is smaller.

Table 1 . The average values of OSPA and running time comparison

\begin{tabular}{|c|c|c|c|}
\hline Average values & RBAP-PHD & AP-PHD & AP-K-MEANS-PHD \\
\hline OSPA[m] & 30.445 & 32.012 & 56.420 \\
\hline Time[s] & 0.501 & 0.489 & 0.510 \\
\hline
\end{tabular}

Table 1 illustrate the average values of OSPA distance over the 50 steps and average running time for each step of the three filters. AP-K-MEANS-PHD performs the worst, and RBAP-PHD improves the accuracy by about $1.6 \mathrm{~m}$ averagely compare to AP-PHD. However, since every particle is processed by KF, its average running time is longer than that of AP-PHD. For obtaining the similar precision, we add the persistent particle number to 220 for AP-PHD. After 100 Monte Carlo runs, the average OSPA value of AP-PHD is $30.475 \mathrm{~m}$, which is close to RBAP-PHD, but its average running time per step increases to 1.018s. On the premise of similar tracking precision, RBAP-PHD saves almost half of the running time relative to AP-PHD.

Fig. 4 shows the cardinality estimation of all three filters. Since their essence is APF and their implementation ways are identical, so their cardinality estimation results are very similar. The three filters can detect and report newborn targets in a very short of time and respond quickly to target death.

\section{Conclusion}

This paper presents an improved free clustering P-PHD filter to enhance the tracking precision. Inspired by the Rao-Blackwell theorem, the new PHD filter combines KF and APF to estimate the linear and non linear components in multi-target state. The simulation result show that state estimation obtained from updated weights of particles is more accurate than clustering particles via K-Means algorithm, and the presented approach also outperforms the existing APF based P-PHD. Though the presented improved free clustering P-PHD filter needs more time to complete tracking when the numbers of particles are identical to APF based P-PHD without clustering step, the latter requires more particles and about twice as much time to reach similar precision. Rao-Blackwellized 
free clustering P-PHD filter increases efficiency of the existing free clustering APF based P-PHD filter without losing the reliability of target number estimation.

\section{Acknowledgements}

This work is supported by National Natural Science Foundation of China (61370037,61005026), Gansu Province Basic Research Innovation Group Project(1506RJIA031), Gansu Provincial Science and Technology Planning of China (1506RJZA090) and Foundation of Higher Education of Gansu Province, China (2014A-035).

\section{References}

[1] Bar-Shalom Y, Willett P K, Tian X. Tracking and data fusion[J]. A Handbook of Algorithms. Yaakov Bar-Shalom, 2011.

[2] Blackman S S. Multiple hypothesis tracking for multiple target tracking[J]. Aerospace and Electronic Systems Magazine, IEEE, 2004, 19(1): 5-18.

[3] Mušicki D, Evans R. Joint integrated probabilistic data association: JIPDA[J]. Aerospace and Electronic Systems, IEEE Transactions on, 2004, 40(3): 1093-1099.

[4] Mahler. Multitarget Bayes filtering via first-order multitarget moments[J]. Aerospace and Electronic Systems, IEEE Transactions on, 2003, 39(4): 1152-1178.

[5] Vo B N, Singh S, Doucet A. Sequential Monte Carlo methods for multitarget filtering with random finite sets[J]. Aerospace and Electronic Systems, IEEE Transactions on, 2005, 41(4): 1224-1245.

[6] Vo B N, Singh S, Doucet A. Sequential Monte Carlo implementation of the PHD filter for multi-target tracking[C]//Proc. Int'l Conf. on Information Fusion. 2003: 792-799.

[7] Sidenbladh H. Multi-target particle filtering for the probability hypothesis density[J]. arXiv preprint cs/0303018, 2003.

[8] Schön T, Gustafsson F, Nordlund P J. Marginalized particle filters for mixed linear/nonlinear state-space models[J]. Signal Processing, IEEE Transactions on, 2005, 53(7): 2279-2289.

[9] Karlsson R, Schön T, Gustafsson F. Complexity analysis of the marginalized particle filter[J]. 2004.

[10] Petetin Y, Desbouvries F. Marginalized PHD Filters for multi-target filtering[C]//Information Science, Signal Processing and their Applications (ISSPA), 2012 11th International Conference on. IEEE, 2012: 419-424.

[11] Karlsson R, Gustafsson F. Recursive Bayesian estimation: bearings-only applications[C]//Radar, Sonar and Navigation, IEE Proceedings-. IET, 2005, 152(5): 305-313.

[12] Särkkä S, Vehtari A, Lampinen J. Rao-Blackwellized particle filter for multiple target tracking[J]. Information Fusion, 2007, 8(1): 2-15.

[13] Clark D E, Bell J. Multi-target state estimation and track continuity for the particle PHD filter[J]. Aerospace and Electronic Systems, IEEE Transactions on, 2007, 43(4): 1441-1453.

[14] Tang X, Wei P. Multi-target state extraction for the particle probability hypothesis density filter[J]. IET radar, sonar \& navigation, 2011, 5(8): 877-883.

[15] Ristic B, Clark D, Vo B N. Improved SMC implementation of the PHD filter[C]//Information Fusion (FUSION), 2010 13th Conference on. IEEE, 2010: 1-8. 